\title{
Assessment of Staffing, Services, and Partnerships of Local Health Departments — United States, 2015
}

\author{
Sarah J. Newman, $\mathrm{MPH}^{1}$; Jiali Ye, $\mathrm{PhD}^{1}$; Carolyn J. Leep, $\mathrm{MPH}^{1}$; LaMar Hasbrouck, $\mathrm{MD}^{1}$; Carlos Zometa, $\mathrm{PhD}^{2}$
}

Beginning in 2008, the National Association of County and City Health Officials (NACCHO) periodically surveyed local health departments (LHDs) to assess the impact of the economic recession on jobs and budgets (1). In 2014, the survey was expanded to assess a wider range of factors affecting programs, services, and infrastructure in LHDs and renamed the Forces of Change survey (2). The survey was administered in to January-February 2015 to 948 LHDs across the United States to assess budget changes, job losses, changes in services, and collaboration with health care partners; 690 (73\%) LHDs responded. Findings indicated a change in LHD infrastructure: compared with the previous fiscal year.* Overall, LHDs reported 3,400 jobs lost; $25 \%$ of LHDs reported budget decreases; $36 \%$ reported a reduction in at least one service area; and 35\% reported serving fewer patients in clinics. In addition, up to $24 \%$ of LHDs reported expanding populationbased prevention services, and LHDs reported exploring new collaborations with nonprofit hospitals and primary care providers (PCPs).

The public health and clinical care environment is evolving in part in response to the Patient Protection and Affordable Care Act (ACA). Section 501(r)(3) of the Patient Protection and Affordable Care Act (ACA) requires that nonprofit hospitals conduct and report on a community health needs assessment (CHNA) every 3 years to maintain their tax-exempt status (3). The ACA also requires that a CHNA take into account input from stakeholders that represent the broad interests of the community served by the hospital, including those with special knowledge or expertise in public health, such as LHDs. New systems of care with PCPs intended to improve patient outcomes and reduce costs have also been developed in recent years. These include State Innovation Models (state-based, multipayer health care payment and service delivery models), patient-centered medical homes (primary care delivery models that are patient-centered, comprehensive, team-based, accessible, and focused on quality and safety), and accountable care organizations (networks of health care providers voluntarily responsible for providing coordinated care to patients) (4).

An online survey was distributed during January-February 2015 to a statistically representative sample of 948 LHDs across all regions of the United States, representing approximately one third of all LHDs. LHDs were stratified by state and size of the population served (small $[<50,000$ persons], medium $[50,000-499,999]$, and large $[>500,000])$. Hawaii and Rhode Island were excluded from the study because they have no LHDs. Survey topics were identified by NACCHO's executive leadership, and several partner organizations provided input on the highest-priority topics, which included changes in LHD budgets, staffing, and services provided from the previous year; changes in clinical service delivery; third-party billing for clinical services; collaboration with nonprofit hospitals; and collaboration with PCPs (5). A survey instrument with 16 closed-ended questions was developed, reviewed by subjectmatter experts, and piloted. Responses were self-reported and were not independently verified by NACCHO. Nationally representative estimates were weighted to account for sampling design and nonresponse. Information about the survey methods is available in the survey's technical documentation (6).

The survey was completed by 690 (73\%) top executives from 353 small, 271 medium, and 66 large LHDs. All surveyed areas except the District of Columbia and Massachusetts achieved a response rate of $\geq 50 \%$ (6). Overall, $23 \%$ of LHDs reported a lower budget in the current fiscal year compared with the preceding fiscal year, and $27 \%$ reported that they expected budget decreases to continue into the next fiscal year (Table). LHDs reported 3,400 jobs lost during 2014 (1,300 [38\%] because of layoffs and 2,100 [62\%] because of attrition). Since 2008 , a total of 51,700 jobs have been lost. The number of lost jobs in 2014 was most marked among large LHDs: 61\% of large LHDs reported at least one job lost, followed by $41 \%$ of medium LHDs and 26\% of small LHDs. Approximately one third (36\%) of LHDs reported reduced services in at least one program area during 2014. More LHDs reported reducing rather than expanding clinical services such as immunization (14\% reducing versus $12 \%$ expanding), diabetes screening (14\% versus $11 \%)$, or high blood pressure screening $(11 \%$ versus $8 \%)$.

Approximately one third (35\%) of LHDs reported serving fewer patients in their clinics during 2014 than 2013 (Table); this varied by state (Figure 1). However, a larger proportion of LHDs reported expanding population-based prevention services: $24 \%$ of LHDs expanded obesity prevention services, and $23 \%$ reported expanding tobacco, alcohol, and other drug prevention services.

\footnotetext{
* Fiscal years vary across LHDs in the United States.
} 
TABLE. Number and percentage of 690 local health departments (LHDs) reporting recent budget changes, job losses, changes in services, third party billing practices, and collaboration with nonprofit hospitals and primary care providers, by size of population served — National Association of County and City Health Officials Forces of Change survey, United States, 2015

\begin{tabular}{|c|c|c|c|c|c|c|}
\hline \multirow[b]{2}{*}{ Factor } & \multirow{2}{*}{$\begin{array}{l}\text { No. of LHDs } \\
\text { responding }\end{array}$} & \multirow{2}{*}{$\begin{array}{c}\text { Unweighted } \\
\text { no. }\end{array}$} & \multicolumn{4}{|c|}{ Size $^{\S}$ of population served by LHD (\%) } \\
\hline & & & All & Small & Medium & Large \\
\hline \multicolumn{7}{|l|}{ Budget changes } \\
\hline Lower budget than the previous fiscal year & 666 & 151 & 23 & 22 & 23 & 25 \\
\hline Expect lower budget in the next fiscal year & 632 & 171 & 27 & 25 & 28 & 33 \\
\hline Higher budget than the previous fiscal year & 666 & 143 & 21 & 17 & 28 & 16 \\
\hline Expect higher budget in the next fiscal year & 632 & 109 & 17 & 15 & 20 & 19 \\
\hline \multicolumn{7}{|l|}{ Job losses in 2014} \\
\hline Lost at least one job because of layoffs and/or attrition & 657 & 227 & 34 & 26 & 41 & 61 \\
\hline \multicolumn{7}{|l|}{ Changes in services provided in 2014} \\
\hline Reduced services in at least one program area & 679 & 251 & 36 & 35 & 38 & 38 \\
\hline Expanded services in at least one program area & 679 & 361 & 53 & 48 & 59 & 58 \\
\hline Reduced immunization services & 657 & 98 & 14 & 14 & 14 & 21 \\
\hline Expanded immunization services & 657 & 82 & 12 & 14 & 12 & 4 \\
\hline Reduced diabetes screening services & 255 & 37 & 14 & 14 & 15 & 15 \\
\hline Expanded diabetes screening services & 255 & 31 & 11 & 6 & 18 & 23 \\
\hline Reduced high blood pressure screening services & 412 & 44 & 11 & 10 & 12 & 15 \\
\hline Expanded high blood pressure screening services & 412 & 36 & 8 & 6 & 14 & 1 \\
\hline Reduced obesity prevention services & 458 & 35 & 7 & 9 & 7 & 6 \\
\hline Expanded obesity prevention services & 458 & 110 & 24 & 17 & 31 & 28 \\
\hline Reduced tobacco, alcohol, and other drug prevention services & 514 & 46 & 9 & 11 & 6 & 11 \\
\hline Expanded tobacco, alcohol, and other drug prevention services & 514 & 118 & 23 & 20 & 27 & 23 \\
\hline \multicolumn{7}{|l|}{ Changes in clinical service delivery in 2014 compared with 2013} \\
\hline Served fewer patients & 626 & 221 & 35 & 34 & 37 & 33 \\
\hline Served the same number of patients & 626 & 269 & 43 & 44 & 40 & 44 \\
\hline Served more patients & 626 & 136 & 22 & 21 & 23 & 24 \\
\hline Served fewer patients with insurance & 662 & 46 & 7 & 7 & 7 & 6 \\
\hline Served the same number of patients with insurance & 662 & 186 & 28 & 29 & 29 & 25 \\
\hline Served more patients with insurance & 662 & 258 & 38 & 37 & 39 & 45 \\
\hline \multicolumn{7}{|l|}{ Current third-party billing for clinical services } \\
\hline Bill public payers only & 610 & 149 & 23 & 21 & 24 & 38 \\
\hline Bill public and private payers & 610 & 428 & 66 & 66 & 69 & 53 \\
\hline Bill private payers only & 610 & 3 & 0.5 & 1 & 0 & 2 \\
\hline Do not bill & 610 & 63 & 10 & 12 & 8 & 7 \\
\hline \multicolumn{7}{|c|}{ Collaboration with nonprofit hospitals on community health needs assessments } \\
\hline Currently collaborating & 621 & 367 & 58 & 49 & 67 & 67 \\
\hline Discussing collaboration & 621 & 59 & 9 & 8 & 8 & 24 \\
\hline Not engaged in discussion or collaboration & 621 & 72 & 12 & 13 & 11 & 9 \\
\hline \multicolumn{7}{|l|}{ Involvement in nonprofit hospital implementation plans } \\
\hline Involved in nonprofit hospital implementation plan & 515 & 313 & 60 & 58 & 61 & 60 \\
\hline Listed as partner in implementation plan & 402 & 402 & 47 & 43 & 49 & 52 \\
\hline Participated in developing the implementation plan & 402 & 168 & 41 & 41 & 43 & 29 \\
\hline Listed as conducting an activity in the implementation plan & 402 & 402 & 20 & 16 & 24 & 21 \\
\hline Used the same implementation plan & 402 & 39 & 10 & 9 & 11 & 5 \\
\hline \multicolumn{7}{|l|}{ Active collaboration with primary care providers (PCPs) } \\
\hline Encouraged PCPs to use evidence-based public health services & 663 & 411 & 61 & 58 & 63 & 76 \\
\hline Provided population health statistics to PCPs & 661 & 316 & 47 & 39 & 54 & 59 \\
\hline Used clinical data from PCPs & 643 & 148 & 23 & 21 & 23 & 32 \\
\hline Participated in State Innovation Model initiative activities & 659 & 66 & 9 & 4 & 14 & 23 \\
\hline Participated in patient-centered medical home activities & 658 & 63 & 9 & 6 & 12 & 19 \\
\hline Participated in accountable care organizations & 657 & 53 & 8 & 7 & 9 & 9 \\
\hline
\end{tabular}

* Hawaii and Rhode Island not included.

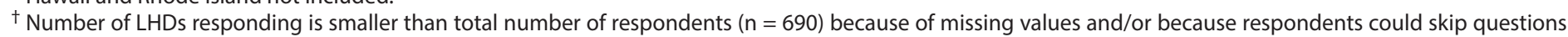
based on their responses to screening questions.

$\S$ Small: serve $<50,000$ persons $(n=353$ LHDs); medium: serve 50,000-499,999 persons $(n=271)$; large: serve $>500,000$ persons $(n=66)$.

During 2014, 38\% of LHDs reported serving a higher percentage of insured patients than they had during 2013. Among sampled LHDs in 26 states that expanded Medicaid eligibility in 2015, 46\% reported serving a higher percentage of patients with insurance, compared with $29 \%$ in states that did not expand Medicaid eligibility. Most LHDs (90\%) bill thirdparty payers (i.e., Medicare, Medicaid, and private insurers) for some services; $66 \%$ of LHDs reported they billed both public 
FIGURE 1. State* percentage of local health departments serving fewer patients in their clinics in 2014 compared with 2013 - United States

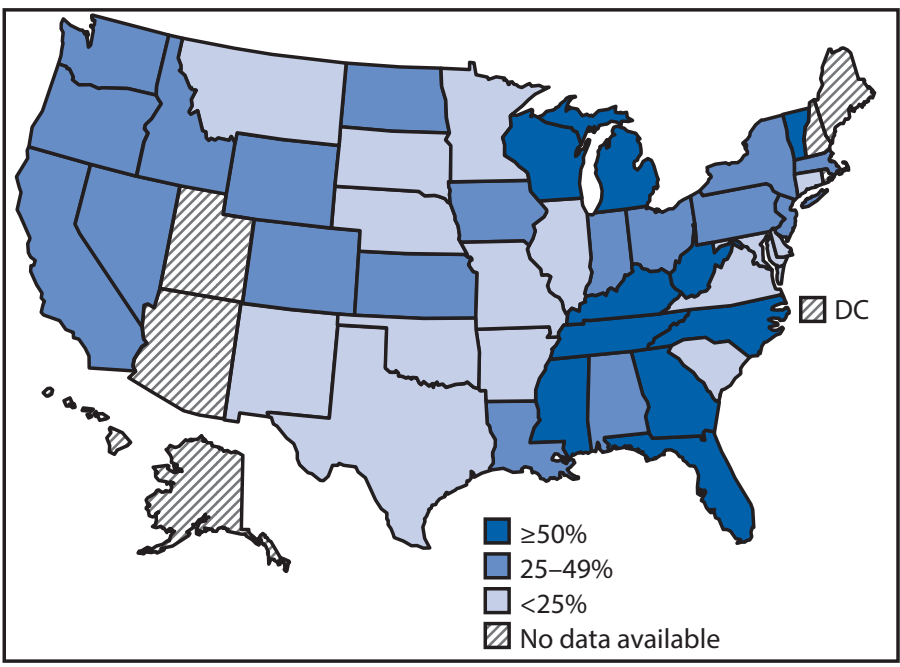

* Hawaii and Rhode Island excluded because they have no local health departments. Data from states with insufficient response rates (Alaska, Arizona, District of Columbia, Maine, New Hampshire, and Utah) not shown.

and private payers for at least some services, and 23\% reported they billed public payers only. Respondents reported that the cost and complexity of establishing billing, the existence of a trained workforce, and information technology capacity were most important in determining billing practices.

Approximately half (58\%) of LHDs reported that they were currently collaborating with nonprofit hospitals to conduct CHNAs. A smaller percentage (9\%) of LHDs were considering future collaboration, and some (12\%) were not engaged in discussions to collaborate. The remaining LHDs (21\%) did not report having a nonprofit hospital serving their jurisdiction. In addition, among LHDs with a nonprofit hospital serving their jurisdiction, $60 \%$ were involved in a nonprofit hospital's implementation plan for the CHNA. Among these, $47 \%$ were listed as a partner in the plan, $41 \%$ participated in developing the plan, $20 \%$ reported that they were conducting an activity in the plan, and $10 \%$ of LHDs reported using the same implementation plan as the hospital.

LHDs also reported collaborating with PCPs. Approximately half (61\%) of LHDs actively encouraged PCPs to use evidence-based public health services, such as interventions to reduce asthma triggers in children's homes; $47 \%$ provided population health statistics to PCPs; and 23\% used clinical data from PCPs. Overall, less than 10\% of LHDs were actively engaged in new systems of care with PCPs including State Innovation Models, patient-centered medical homes, or accountable care organizations. This engagement also varied across states (Figure 2).
FIGURE 2. State* percentage of local health departments actively engaged with primary care providers on State Innovation Models, accountable care organizations, or patient-centered medical homes United States, 2014

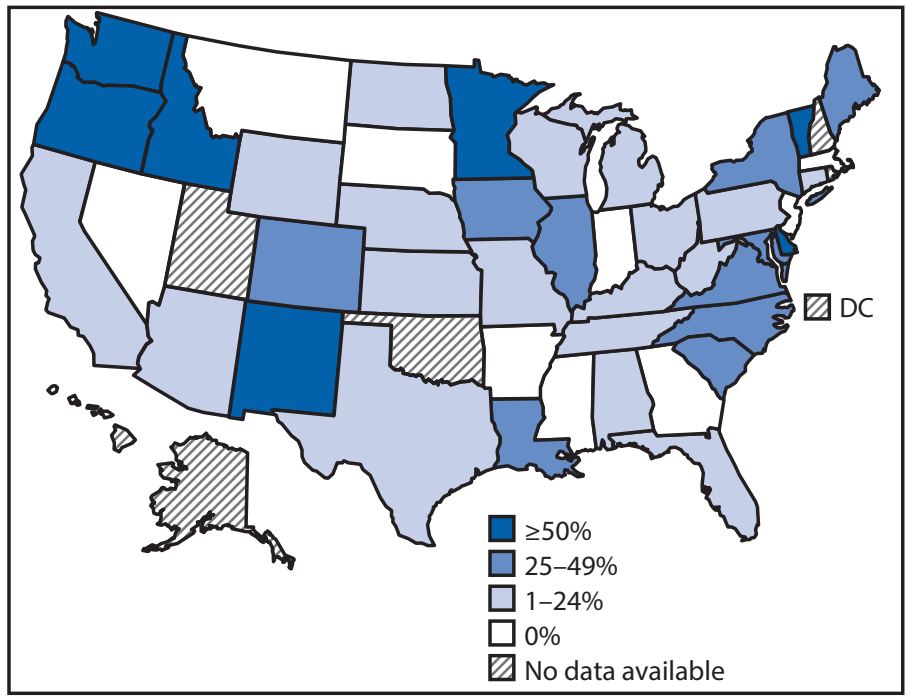

* Hawaii and Rhode Island excluded because they have no local health departments. Data from states with insufficient response rates (Alaska, District of Columbia, New Hampshire, Oklahoma, and Utah) not shown.

\section{Discussion}

The severe United States economic recession (December 2007June 2009) substantially affected the operating budgets of LHDs. Although the proportion of LHDs reporting budget decreases in the past year has decreased from its peak of $45 \%$ in 2009 (1), approximately one in four LHDs still reported budget cuts in the current fiscal year compared to the previous fiscal year. Since 2008, LHDs have collectively lost 51,700 jobs because of layoffs and attrition (1). For many LHDs, the cumulative effects of budget cuts and job losses experienced during and after the recession have not been reversed as the economy recovered. Consequently, the cumulative effects of years of budget cuts and job losses continue to reduce capacity at many LHDs and decrease the ability of LHDs to prepare for the future.

The ACA's expansion of insurance benefits is reflected in changes in patient volume at LHDs and percentage of patients at LHDs who have insurance. More LHDs reported a decrease in patient volume than an increase in patient volume, and LHDs reported serving higher percentages of patients with insurance, although neither trend has been uniform across the United States. Patients who have insurance might preferentially seek services at other sources of health care than the LHD. This might present an opportunity for LHDs to create new and expand existing partnerships. With the exception of a few states, LHDs are not currently engaged in new systems of care 


\section{Summary}

What is already known about this topic?

The public health and clinical care environment is evolving in response to the Patient Protection and Affordable Care Act.

\section{What is added by this report?}

Local health department (LHD) infrastructure continues to be affected by budget decreases: one quarter of LHDs reported a lower budget in the current fiscal year compared to the previous fiscal year. LHDs reported 3,400 fewer jobs in 2014 than in 2013 and 51,700 jobs lost since $2008 ; 36 \%$ of LHDs reported a reduction in at least one service area, and $35 \%$ reported serving fewer patients in clinics. Up to $24 \%$ of LHDs reported expanding population-based prevention services, and LHDs reported they are exploring new collaborations with nonprofit hospitals and primary care providers.

What are the implications for public health practice?

Ongoing budget cuts and resulting personnel layoffs jeopardize the work of LHDs, which remain primary providers of health care services for many clients. As shown through their new collaborations with nonprofit hospitals and exploration of relationships with primary care providers, LHDs continue to build and explore critical local relationships that might benefit multiple stakeholders and their communities at large.

established by the ACA, such as accountable care organizations or State Innovation Models. The ACA requirement for nonprofit hospitals to complete regular CHNAs provides an opportunity for LHDs to collaborate with nonprofit hospitals. Less than $70 \%$ of LHDs are engaged in or exploring such partnerships, which might benefit multiple stakeholders and the community at large.

The findings in this report are subject to at least three limitations. First, the survey instrument includes only closed-ended questions about a limited number of topics. Consequently, other important factors not addressed by this survey might be affecting change in LHDs. Second, only descriptive statistics were presented, and no conclusions can be drawn about cause and effect. Finally, all data were self-reported by LHDs and not verified by NACCHO; therefore, the data are subject to reporting errors that cannot be identified or quantified.
LHDs face challenges and opportunities as the new public health and clinical care environments evolve. Some LHDs are adapting by reducing clinical services or expanding populationbased prevention services; others continue to sustain clinical services by expanding reimbursement for those services through billing third-party payers. The ACA has also presented new opportunities for collaboration, and many LHDs are engaged in or exploring these new partnerships. Given the variations in LHD capacity to adapt to budget cuts, job losses, and reductions in clinical services while simultaneously having to implement their vision of healthy communities, LHDs will need to adopt diverse roles within their local public health systems ( 7 ).

\footnotetext{
${ }^{1}$ National Association of County and City Health Officials, Washington, D.C ${ }^{2}$ Office of State, Tribal, Local and Territorial Support, CDC.

Corresponding author: Sarah J. Newman, snewman@naccho.org, 202-640-4923.
}

\section{References}

1. Ye J, Leep C, Newman S. Reductions of budgets, staffing, and programs among local health departments: results from NACCHO's economic surveillance surveys, 2009-2013. J Public Health Manag Pract 2015;21:126-33. http://dx.doi.org/10.1097/PHH.0000000000000074

2. Newman S, Leep C, Ye J, Robin N. The changing public health landscape: findings from the 2015 forces of change survey. Washington DC: National Association of County and City Health Officials; 2015. http:// nacchoprofilestudy.org/wp-content/uploads/2015/04/2015-Forces-ofChange-Slidedoc-Final.pdf

3. Internal Revenue Service. New requirements for 501(c) (3) hospitals under the Affordable Care Act. Washington, DC: US Department of Treasury, Internal Revenue Service; 2016. https://www.irs.gov/charities-non-profits/charitable-organizations/ new-requirements-for-501c3-hospitals-under-the-affordable-care-act

4. Centers for Medicare \& Medicaid Services. Innovation models. Baltimore, MD: US Department of Health and Human Services, Centers for Medicare \& Medicaid Services; 2016. http://innovation.cms.gov/initiatives

5. National Association of County and City Health Officials. Forces of change. Methods. 2015 forces of change survey instrument. Washington DC: National Association of County and City Health Officials; 2015. http://nacchoprofilestudy.org/forces-of-change

6. National Association of County and City Health Officials. 2015 Forces of change. Technical documentation. Washington DC: National Association of County and City Health Officials; 2015. http:// nacchoprofilestudy.org/wp-content/uploads/2015/04/Forces-of-ChangeTechnical-Documentation-Final.pdf

7. Public Health Leadership Forum. The high achieving governmental health department in 2020 as the community chief health strategist. http://www. resolv.org/site-healthleadershipforum/files/2014/05/The-HighAchieving-Governmental-Health-Department-as-the-Chief-HealthStrategist-by-2020-Final1.pdf 